\section{(6) OPEN ACCESS}

\title{
Can initial clinical assessment exclude thoracolumbar vertebral injury?
}

\author{
Dinendra Singh Gill, ${ }^{1}$ Biswadev Mitra, ${ }^{2,3}$ Fairleigh Reeves, ${ }^{2}$ Peter A Cameron, ${ }^{2,3,4}$ \\ Mark Fitzgerald, ${ }^{4,5}$ Susan Liew, ${ }^{5,6}$ Dinesh Varma ${ }^{7}$
}

\begin{abstract}
- Additional supplementary files are published online only. To view these files please visit the journal online (http://dx.doi. org/10.1136/emermed-2011201085)
\end{abstract}

${ }^{1}$ Emergency Department, Morriston Hospital,

Swansea, UK

${ }^{2}$ Emergency \& Trauma Centre,

The Alfred Hospital, Melbourne, Australia

${ }^{3}$ Department of Epidemiology \& Preventive Medicine, Monash University, Melbourne, Australia ${ }^{4}$ National Trauma Research Institute, Melbourne, Australia

${ }^{5}$ Trauma Service, The Alfred Hospital, Melbourne, Australia ${ }^{6}$ Department of Surgery, Monash University, Melbourne, Australia

${ }^{7}$ Department of Radiology, The Alfred Hospital, Melbourne, Australia

Correspondence to Dr Dinendra Singh Gill, Emergency Department, Morriston Hospital (ABM University Health Board), Heol Maes Eglwys, Morriston, Swansea SA6 6NL, UK; dinendra.gill@wales.nhs.uk or dsg741@gmail.com

Accepted 2 August 2012 Published Online First 22 August 2012

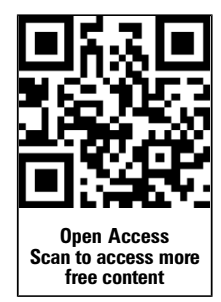

\begin{abstract}
Introduction The aim of this study was to test the hypothesis that all blunt trauma patients, presenting with a Glasgow coma scale (GCS) score of 15, without intoxication or neurological deficit, and no pain or tenderness on log-roll can have any thoracolumbar fracture excluded without imaging.
\end{abstract}

Materials and Methods All patients diagnosed with a thoracolumbar fracture presenting to the emergency department of a major trauma centre and having an initial GCS score of 15 were included in the study. Variables collected included type of fracture, mechanism of injury, the presence of pain or tenderness on log-roll, ethanol levels and prehospital opioid analgesia.

Results There were 536 patients with thoracolumbar fractures, of which 508 (94.8\%) patients had either pain, tenderness or had received prehospital opioid analgesia. A small subgroup of 28 (5.2\%) patients who received no prehospital opioid analgesia, did not complain of pain and had no tenderness to the thoracolumbar spine elicited on log-roll. This subgroup was significantly older $(p=0.033)$ and a high proportion of patients (64.3\%) had a concurrent fracture of the cervical spine. Within this subgroup, a clinically significant unstable thoracic fracture was present in three patients, with all three patients exhibiting symptoms and signs of neurological injury or having a concurrent cervical vertebral fracture.

Conclusions In this population of blunt trauma patients with a GCS score of 15 , not under the influence of alcohol or prehospital morphine administration, the absence of pain or tenderness on log-roll can exclude a clinically significant lumbar vertebral fracture, but does not exclude a thoracic fracture.

\section{INTRODUCTION}

Early and accurate assessment of the thoracolumbar spine is an important aspect of trauma reception and resuscitation. Between 19\% and 50\% of fractures may have associated neurological damage to the spinal cord. ${ }^{1}$ Missed or delayed diagnosis can lead to a multitude of problems including long-term pain, reduced quality of life, and can have devastating psychological effects.

It is widely recognised that blunt trauma patients with altered mental status require imaging of the cervical and thoracolumbar spine, as clinical signs and symptoms can be unreliable. ${ }^{2-6}$ In patients with a Glasgow coma scale (GCS) score of 15 , much work has been done with regard to clearing the cervical spine of significant injury with established and validated gudelines. ${ }^{7} 8$ To date, there are only a few small prospective studies 56910 and a number of small retrospective analyses directed at the assessment of the thoracolumbar spine. $.^{2-4} 11-14$ Differences in clinical anatomy make direct translation of the results from studies on the cervical spine unreliable. The greater mass, longer distance from spinous processes to anterior body and relative immobility of the thoracolumbar vertebrae compared to the cervical vertebrae are key differences.

Despite these differences in a recent systematic review it has been proposed that those patients who are awake, without evidence of intoxication, with normal mental, neurological and physical examinations can be cleared clinically. ${ }^{15}$ The aim of this study was to test this hypothesis by looking at patients presenting with thoracolumbar fractures with a GCS score of 15.

\section{METHODS}

\section{Setting}

The Alfred Hospital is one of two adult tertiary trauma referral centres in Melbourne, Australia, and serves the statewide population of Victoria of approximately five million. It has an annual emergency department (ED) census of over 45000 patients, with more than 1200 major trauma (injury severity score (ISS) >15) admissions per annum.

The diagnostic imaging evaluation of the thoracolumbar spine of patients presenting to the emergency and trauma centre includes anteroposterior and lateral views of the thoracic and lumbar spine. Patients with a higher suspicion of fracture or those with pain or tenderness undergo CT scanning of the thoracic and/or lumbar spine. Patients already having CT scanning of the chest or abdomen have reformatted images of the thoracic and lumbar spine developed without additional scanning. The CT scanner used was a GE Lightspeed VCT 64-slice scanner (General Electrical Company, GE Healthcare, Milwaukee, USA).

\section{Patients}

The Alfred Trauma Registry, funded as part of the Victorian State Trauma System, collects trauma data concurrent with the inpatient episode. Data are collected according to a defined dataset by experienced registry staff and regularly audited. The registry collects data on all patients admitted for more than $24 \mathrm{~h}$ to the Alfred Trauma Service, trauma patients with an ISS of more than 15 and patients admitted for over $72 \mathrm{~h}$ post-injury admitted under other units. Patients with ISS less than 15 and isolated trauma to the vertebrae were therefore included, provided they spent over $72 \mathrm{~h}$ in hospital. All patients diagnosed with a thoracolumbar fracture presenting to the ED between January 2006 and December 2008 were identified from the 
Alfred trauma database and included in the this study. Patients presenting for elective procedures and follow-up were excluded.

\section{Study design}

Data collected from the trauma registry included patient demographics, mechanism of injury, the first recorded GCS score on arrival to the ED, abbreviated injury scale codes with descriptions and ISS. A subgroup of patients with an initial GCS score of 15 was identified for analysis. A retrospective explicit chart review of these patient records was conducted by DSG and FR and audited by a third operator (BM). Any records with ambiguous, missing or unknown data were reviewed by all three operators and discussed at study coordination meetings held at regular intervals. Variables for collection were defined before the chart review and objectively coded when possible. Thoracolumbar pain was coded as a symptom if mentioned in either the initial assessment notes of ambulance personnel, nursing or medical staff. Data on prehospital analgesia given and finding of tenderness on log-roll during secondary survey were gathered from chart reviews, while blood alcohol levels were obtained from the Alfred pathology service. All variables were documented in predetermined abstraction forms.

\section{Analysis}

Continuous data are presented as mean with SD, whereas ordinal data are presented as medians with interquartile ranges. All analysis was performed using SAS V.8.2. Sensitivities were calculated for clinical features used in the assessment of thoracolumbar fractures. Student's t test was used to calculate the significance between two continuous variables, whereas the Wilcoxon rank sum test was used to compare ordinal variables. All p values are reported at the $95 \%$ CI.

The study was approved by the Alfred Hospital Research and Ethics Committee.

\section{RESULTS}

There were 1161 patients with thoracolumbar fractures over the study period, with a total of 1902 fractures. Exclusion criteria are presented in figure 1 with 536 patients included for analysis. The average age was $44.3 \pm 18.6$ years with a male to female ratio of $2.8: 1$ and a median ISS of 16 (9-22).

Of the 536 patients presenting to ED with a GCS score of $15,354(66.0 \%)$ patients received prehospital opioid analgesia. The presence of thoracolumbar pain was documented in 325 $(60.6 \%)$ on initial assessment, while tenderness on log-roll was elicited in $323(60.3 \%)$ patients. There were 52 (9.7\%) patients with a positive blood alcohol test. A positive blood alcohol

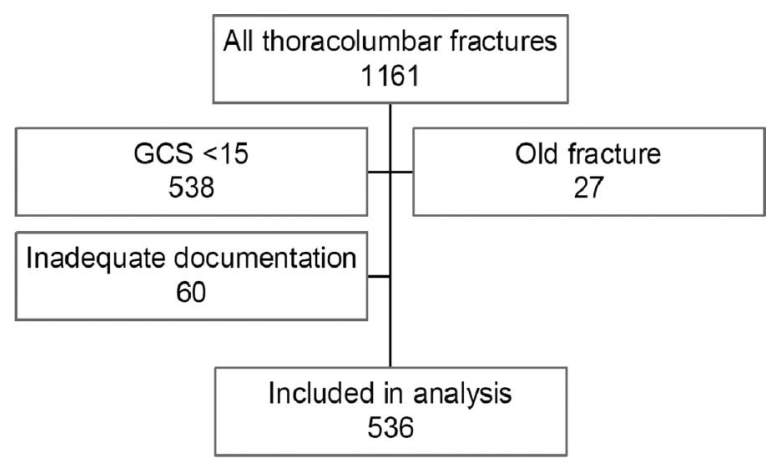

Figure 1 Inclusions and exclusions. GCS, Glasgow coma scale score. level was defined as any value greater than $0 \mathrm{~g}$ per $100 \mathrm{ml}$ blood.

The sensitivities of prehospital opioid analgesia, pain to thoracolumbar area, tenderness on log-roll and a positive blood alcohol, together with a combination of all variables are presented in table 1 . Sensitivities subgrouped by mechanism of injury are presented in table 2 .

All patients with a lumbar vertebral fracture complained of pain and/or received prehospital analgesia and/or had tenderness on log-roll examination. There were 28 (5.2\%) patients who were diagnosed with a thoracic vertebral fracture, but received no prehospital opioid analgesia, did not complain of pain on initial presentation and had no tenderness to the thoracolumbar spine elicited on log-roll. The blood alcohol level was not measured in 15 of these patients. The median ISS in this group of patients was 13.5 (9-19), which was not significantly different to the ISS for the overall group $(p=0.252)$. This patient subgroup was significantly older at $52.3 \pm 21.9$ years $(p=0.033)$. There were seven patients who sustained their injury after a low fall $(<1 \mathrm{~m})$, three patients after a high fall $(>1 \mathrm{~m})$, seven motor vehicle crashes, six motorcycle crashes, three bicycle related and two pedestrians. Of these 28 patients, there were 18 patients with a concurrent fracture of the cervical spine, four (14\%) had rib fractures and six (22\%) had other non-spinal fractures. Tertiary survey of these patients revealed tenderness to the thoracolumbar spine in only two cases, both of whom had concurrent cervical spine fractures.

Non-significant fractures were defined as involving only one column (stable fractures) and not requiring operative fixation. Of the above group, 25 (89\%) patients sustained non-significant stable fractures including 10 anterior compression fractures, eight superior end plate fractures, four transverse process fractures, one spinous process fracture and two other minor abnormalities.

The remaining three $(11 \%)$ patients sustained clinically significant fractures defined as those involving two or more columns (unstable fractures according to the Denis classification $)^{16}$ or those requiring operative fixation. This included a 25-year-old motorcyclist sustaining a T5 on T6 fracture dislocation, with cord compression, requiring operative fixation. This patient had neurological signs consisting of a T5 sensory level and paraplegia. There was also a 75-year-old patient with a three column fracture from a fall from standing height and a 33-year-old pedal cyclist with a two column fracture. Neither required operative fixation. Both had concurrent cervical vertebral fractures.

\section{DISCUSSION}

This study shows that history and examination could exclude clinically significant fractures of the lumbar vertebrae but could not exclude all fractures of the thoracic vertebrae. In major trauma patients, we have shown that it is possible to exclude 'clinically significant' thoracic fractures based on history and

Table 1 Sensitivity of clinical features for thoracolumbar fractures

\begin{tabular}{lccc}
\hline & $\mathbf{n}^{*}$ & Positive (sensitivity) & $\mathbf{9 5 \%} \mathbf{~ C l}$ \\
\hline Prehospital Morphine & 504 & $354(70.2 \%)$ & 66.0 to 74.1 \\
Pain & 511 & $325(63.6 \%)$ & 59.2 to 69.7 \\
Tenderness & 497 & $323(64.9 \%)$ & 60.6 to 69.1 \\
Blood alcohol & 385 & $52(13.5 \%)$ & 10.3 to 17.4 \\
Any one of above & 536 & $508(94.8 \%)$ & 92.4 to 96.4
\end{tabular}

*Number of patients with documentation. 
Table 2 Sensitivity $(95 \% \mathrm{Cl})$ of clinical variable subgrouped by mechanism of injury

\begin{tabular}{lllllrr}
\hline & $\mathbf{n}$ & Prehospital opioid & Pain & Tenderness & Blood alcohol \\
\hline Low fall & 52 & $75.0(62.8$ to 84.4$)$ & $73.3(57.8$ to 84.9$)$ & $68.1(52.3$ to 80.9$)$ & $7.7(0.4$ to 37.9$)$ & $20.5(12.3$ to 31.9$)$ \\
High fall & 109 & $75.3(65.0$ to 83.3$)$ & $77.3(67.5$ to 84.9$)$ & $70.4(59.6$ to 79.4$)$ & $97.5(74.0$ to 94.8$)$ \\
MVA & 174 & $72.7(64.5$ to 79.7$)$ & $54.0(45.3$ to 62.5$)$ & $61.3(52.6$ to 69.4$)$ & $18.8(12.5$ to 27.1$)$ & $95.0(93.7$ to 99.9$)$ \\
MBA & 59 & $76.4(67.0$ to 83.9$)$ & $59.1(49.3$ to 68.2$)$ & $61.5(51.4$ to 70.8$)$ & $6.5(2.7$ to 14.2$)$ & $88.0(75.9$ to 98.3$)$ \\
Pedestrian & 37 & $85.2(65.4$ to 95.1$)$ & $53.6(34.2$ to 72.0$)$ & $60.7(40.7$ to 77.9$)$ & $13.0(3.4$ to 34.7$)$ \\
Bicycle & 34 & $60.9(38.7$ to 79.5$)$ & $46.1(27.1$ to 66.2$)$ & $56.0(35.2$ to 75.0$)$ & $5.0(0.3$ to 26.9$)$ & $96.4(79.8$ to 99.8$)$ \\
Other & 71 & $66.2(53.6$ to 76.9$)$ & $75.0(62.8$ to 84.4$)$ & $72.1(59.7$ to 81.9$)$ & $7.1(1.9$ to 20.5$)$ & $99.6(71.5$ to 97.2$)$ \\
\hline
\end{tabular}

MBA, motorbike accident; MVA, motor vehicle accident.

clinical examination in a subgroup of patients. This subgroup was defined by blunt trauma with a GCS score of 15, the absence of documented alcohol or prehospital morphine, the absence of pain on history and tenderness on log-roll, the absence of neurology and the absence of a cervical spine fracture.

A very small proportion of patients $(4.7 \%)$ were found to have a thoracic fracture in the presence of the above criteria, but were limited to those with a single column injury and none required no operative management. Painless thoracic vertebral fractures have previously been described, ${ }^{17}$ and functional assessment using axial loaded movements have been proposed to determine clinical significance. This subgroup may be allowed to sit up and mobilise. We suggest that should these patients subsequently develop pain, they should be re-examined and have imaging to exclude stable spine injury.

The most devastating clinical consequence of a missed or delayed diagnosis of a thoracolumbar vertebral fracture is the onset and progression of neurological deficits, ${ }^{18} 19$ due to movement at the fracture site, soft tissue swelling, or the development of an epidural haematoma secondary to prophylactic anticoagulation. However, the risk of this occurring has not been clearly quantified in the literature. There is also the potentially positive impact of diagnostic certainty on recovery, rehabilitation, workers compensation, and psychological wellbeing, which requires further clarification. Accurate diagnosis of all injuries remains the ultimate aim during the initial assessment of injured patients.

There is currently some evidence suggesting a mortality benefit when all major trauma patients are analysed. ${ }^{20}$ However, the use of extensive radiography is time consuming, expensive and results in unnecessary radiation exposure, with a potential long-term increased risk of radiation-induced carcinogenesis. The benefit of whole-body CT to trauma patients based on mechanism alone, who exhibit minimal clinical symptoms and signs, remains unknown. Adopting a clinical practice of whole-body CT in this subset of patients exposes them to the long-term risks of ionising radiation and is unlikely to be associated with significant benefit. Obtaining a history and a thorough clinical examination should still play a central role in the assessment of trauma patients.

In our group of patients, the primary difference in excluding thoracolumbar vertebral fractures compared to those of the cervical vertebrae is in the early mobilisation of patients posthistory and examination. While the cervical vertebrae may be mobilised effectively in an awake supine patient, axial loading of thoracolumbar vertebrae through mobilisation is the most effective clinical manoeuvre to detect any pain from a stable fracture. Second, the presence of a cervical spine fracture has previously been shown to be associated with another spinal fracture $^{21}$ and this was confirmed in this study, necessitating imaging of the rest of the spine. The presence of any neurological deficit without pain is a further obvious variable, which necessitates imaging of the thoracolumbar vertebrae.

Contrary to our findings, a negative physical examination alone has previously been reported to be reliable at excluding injury. Samuels and Kerstein $^{12}$ retrospectively reviewed 99 charts, in which 15 patients had thoracolumbar fractures. Of the 55 charts in which patients had no pain or tenderness; there were no missed fractures. However, that series did not report how severely injured patients were and disregarded other factors, which may influence the decision to image the thoracolumbar spine.

The findings of our study are similar to others recommending routine imaging of the thoracolumbar spine. Frankel et $\mathrm{al}^{9}$ found that $40 \%$ of 65 patients with fractures had pain or tenderness. These patients had associated injuries and high blood ethanol levels, making it hard to determine the exact reason for the absence of clinical features. Cooper et $\mathrm{al}^{2}$ reported a review of 183 fractures in 110 patients who were neurologically intact and had a GCS score of 13-15. About a third of these patients had no pain or tenderness, yet all had fractures. The absence of clinical findings was significantly related to the presence of another major injury, defined as abbreviated injury scale scores of 3 or more. Comparison with the findings of our study is difficult, due the small number of patients reviewed and the inclusion of patients with GCS scores of less than 15 in these studies.

To date, this study is the largest retrospective analysis of thoracolumbar vertebral fractures. However, because it is a retrospective review it has limitations and potential bias. Inclusion criteria to the trauma registry excluded those patients who were discharged within $24 \mathrm{~h}$ and those less severely injured. Patients with clinically significant thoracolumbar spine fracture are unlikely to be included in the discharged group. With regard to missing data, very few patients did not have findings of pain on log-roll documented. Using clinical assessment to determine imaging will 'miss' some thoracic vertebral fractures, but the clinical significance of these fractures is likely to be minimal.

The ideal technique of assessing the thoracolumbar spine on 'log-roll' remains unclear. ${ }^{15}$ Being a retrospective review in a large trauma centre, there was a high likelihood of variation in technique for examination ranging from gentle palpation to percussion. This would clearly impact on the presence or absence of tenderness on log-roll. However, it has previously been noted that the 'log-roll' procedure is a team effort and it would be obvious to team members if pain or tenderness were present. ${ }^{15}$ Furthermore, only two patients had tenderness elicited on tertiary survey following a non-tender initial examination, suggesting a measure of agreement.

The spectrum of patients presenting to a large trauma centre is likely to be different to a community hospital. Although 
laboratory alcohol levels would not be available immediately, intoxication could be assessed initially based on clinical suspicion or breath alcohol levels. Blood alcohol level is a routine test performed on all patients who meet trauma call-out criteria. However, for other patients, it is at clinician discretion. Subjective comments regarding alcohol were not considered. We can safely conclude that in patients in whom blood alcohol was not measured or was negative; a GCS score of 15 with history and clinical examination could exclude a clinically significant thoracolumbar spine fracture. If alcohol or non-alcohol intoxicant variables were available for all patients, it would further improve the sensitivity of the rule.

The effect of a 'distracting injury' on the assessment of the thoracolumbar spine cannot be determined from this retrospective review. Terregino et al ${ }^{6}$ looked at 183 clinically evaluable patients, of whom 17 had thoracolumbar fractures, whose only symptoms predictive of a fracture were pain and tenderness. They reported that distracting injury was not predictive of injury.

\section{CONCLUSION}

It is possible to exclude a clinically significant lumbar vertebral fracture post-history and examination in a defined group of major trauma patients, but not a thoracic vertebral fracture. A 'clinically significant' thoracic fracture may be excluded in patients with a GCS score of 15, not under the documented influence of alcohol or prehospital morphine, the absence of pain or tenderness on log-roll, the absence of neurology, and the absence of a concurrent cervical vertebral fracture. These patients do not require imaging and may be mobilised early. Prospective studies are needed to develop algorithms for evaluating the thoracolumbar vertebrae in blunt trauma, based on the needs and aims of individual trauma systems.

Acknowledgement The authors wish to express sincere appreciation to engineer Reza Elmi Lighvan to help in this project.

Contributors BM and PAC were responsible for study design and ethics approval. DSG (first author) was responsible for data procurement, analysis and manuscript production and submission

Funding BM and PAC are supported by grants from the National Health and Medical Research Council, Commonwealth of Australia. No external funding was required for this study.

Competing of interests None.

Ethics approval The study was approved by the Alfred Hospital Research and Ethics Committee.
Provenance and peer review Not commissioned; externally peer reviewed.

Open Access This is an Open Access article distributed in accordance with the Creative Commons Attribution Non Commercial (CC BY-NC 3.0) license, which permits others to distribute, remix, adapt, build upon this work non-commercially, and license their derivative works on different terms, provided the original work is properly cited and the use is non-commercial. See: http://creativecommons.org/licenses/by-nc/3.0/

\section{REFERENCES}

1. Saboe LA, Reid DC, Davis LA, et al. Spine trauma and associated injuries. J Trauma 1991;31:43-9.

2. Cooper C, Dunham C, Rodriguez A. Fall and major injuries are risk factors for thoracolumbar fractures: cognitive impairment and multiple injuries impede the detection of back pain and tenderness. J Trauma 1995;38:692-6.

3. Durham RM, Luchtefeld WB, Wibbenmeyer L, et al. Evaluation of the thoracic and lumbar spine after blunt trauma. Am J Surg 1995;170:681-5

4. Meldon SW, Moettus LN. Thoracolumbar spine fractures: clinical presentation and the effect of altered sensorium and major injury. J Trauma 1995;39:1110-14.

5. Sava J, Williams MD, Kennedy $\mathrm{S}$, et al. Thoracolumbar fracture in blunt trauma: is clinical exam enough for awake patients? J Trauma 2006;61:168-71.

6. Terregino CA, Ross SE, Lipinski MF, et al. Selective indications for thoracic and lumbar radiography in blunt trauma. Ann Emerg Med 1995;26:126-9.

7. Hoffman J, Mower W, Wolfson A, et al. Validity of a set of clinical criteria to rule out injury to the cervical spine in patients with blunt trauma. N Engl J Med 2000;343:94-9.

8. Stiell IG, Wells GA, Vandemheen $\mathrm{KL}$, et al. The Canadian c-spine rule for radiography in alert and stable trauma patients. JAMA 2001;286:1841-8.

9. Frankel HL, Rozycki GS, Ochsner MG, et al. Indications for obtaining surveillance thoracic and lumbar radiographs. J Trauma 1994;37:673-8.

10. Holmes JF, Panacek EA, Miller PQ, et al. Prospective evaluation of criteria for obtaining thoracolumbar radiographs in trauma patient. J Emerg Med 2003;24:1-7.

11. Hsu JM, Joseph T, Ellis AM. Thoracolumbar fracture in blunt trauma patients: guidelines for diagnosis and imaging. Injury 2003;34:426-33.

12. Samuels LE, Kerstein MD. 'Routine' radiologic evaluation of the thoracolumbar spine in blunt trauma patients: a reappraisal. J Trauma 1993;34:85-9.

13. Stanislas MJC, Latham JM, Porter KM, et al. A high risk group for thoracolumbar fractures. Injury 1998;29:15-18

14. Tamir E, Anekstein $Y$, Mirovsky $Y$, et al. Thoracic and lumbar spine radiographs for walking trauma patients - is it really necessary? J Emerg Med 2006;31:403-5.

15. Diaz JJ, Cullinane DC, Altman DT, et al. Practice guidelines for the screening of thoracolumbar spine fracture. J Trauma 2007;63:709-18.

16. Denis $\mathbf{F}$. The three column spine and its significance in the classification of acute thoracolumbar spinal injuries. Spine 1983;8:817-31.

17. Silverman SL. The clinical consequences of vertebral compression fracture. Bone 1992;13(Suppl. 2):S27-31.

18. Anderson S, Biros MH, Reardon RF. Delayed diagnosis of thoracolumbar fractures in multiple trauma patients. Acad Emerg Med 1996;3:832-9.

19. Cummings SR, Melton LJ. Epidemiology and outcomes of osteoporotic fractures. Lancet 2002:359:1761-7.

20. Huber-Wagner S, Lefering R, Qvick LM, et al. Effect of whole-body CT during trauma resuscitation on survival: a retrospective, multicentre study. Lancet 2009;373:1455-61.

21. Singh R, Taylor DM, D'Souza D, et al. Injuries significantly associated with thoracic spine fractures: a case control study. EMA 2009;21:419-23. 